\title{
Healthy and Patient Type 2 Innate Lymphoid Cells are Differently Affected by in vitro Culture Conditions
}

\author{
Maryline Falquet $\mathbb{D}^{1,2}$ \\ Giuseppe Ercolano ${ }^{1,2}$ \\ Peter Jandus ${ }^{3}$ \\ Camilla Jandus ${ }^{1,2, *}$ \\ Sara Trabanelli ${ }^{1,2, *}$
}

'Ludwig Institute for Cancer Research, Lausanne Branch, Lausanne, Switzerland;

${ }^{2}$ Department of Pathology and Immunology, Faculty of Medicine, University of Geneva, Geneva,

Switzerland; ${ }^{3}$ Division of Immunology and

Allergology, University Hospital and

Medical Faculty, Geneva, Switzerland

*These authors contributed equally to this work
Correspondence: Sara Trabanelli

Department of Pathology and

Immunology, University of Geneva, I21 I,

Rue Michel-Servet I, Geneva 4, CH-I2II,

Switzerland

Email sara.trabanelli@gmail.com
Background: Type 2 innate lymphoid cells (ILC2s) have emerged as key players in the development of type 2 driven diseases such as allergy and asthma. Due to their low number in the circulation, in vitro expansion is needed to unravel their mechanisms of action.

Purpose: The aim of this study is to assess the impact of different culture conditions and address whether the method of expansion may distinctly affect healthy donor or patientderived ILC2s.

Methods: Here, we described the impact of six different culture conditions on the proliferation, phenotype and function of human ILC2s freshly obtained from healthy donors (healthy ILC2s) and allergic patients (patient ILC2s).

Results: We showed that the cytokine cocktail or the PHA induced the highest proliferation of healthy ILC2s and patient ILC2s, respectively. We observed that the stromal cells OP9, used as ILC2 feeders, did not boost their proliferation, but impaired the activation marker expression and the function of patient ILC2s. Furthermore, we demonstrated that the culture conditions differently impacted the activation state of $\mathrm{c}-\mathrm{Kit}^{\text {high }}$ and c-Kit ${ }^{\text {low }}$ ILC2s, in both healthy donors and allergic patients. Last, we also observed that ILC2s expanded only with IL-2 and IL-7 were the most prone to secrete IL-5 and IL-13 upon IL-33 stimulation. In contrast, in patients, the addition of OP9 cells during the expansion restrained their type 2 cytokine secretory functions.

Conclusion: This report highlights that culture conditions distinctly impacted on the healthy or patient ILC2 behavior, with important consequences for their study in disease settings.

Keywords: type 2 innate lymphoid cells, in vitro expansion, allergic patients, cytokines

\section{Introduction}

Allergic diseases, including atopic dermatitis/eczema, allergic rhinitis/hay fever, food allergy/ anaphylaxis and asthma, are mainly characterized by a type 2 polarization. In the past years, the prevalence of allergic disease has considerably increased with an estimated prevalence of $30 \%$ to $40 \%$ of the world's population. ${ }^{1}$ Besides known immune cell players such as eosinophils and CD4 Th2 cells, in the last few years, increasing interest in allergy research has focused on innate lymphoid cells (ILCs). ${ }^{2}$ In particular, group 2 innate lymphoid cells (ILC2s) were shown to play a critical role in inducing strong type 2 immune responses in asthma and allergy. ${ }^{3-6}$

Phenotypically, ILC2s are lineage-negative cells, express the transcription factor GATA $3^{7}$ and, in humans, they are defined by the expression of the prostaglandin D2 
receptor $\mathrm{CRTH} 2{ }^{8}$ and variable levels of the stem cell factor receptor c-Kit (CD117). ${ }^{9}$ According to the c-Kit expression, ILC2s can be divided in two distinct populations, the $\mathrm{c}-\mathrm{Kit}^{\text {high }}$ and c-Kit ${ }^{\text {low }}$ ILC2s. ${ }^{10}{ }^{c}-\mathrm{Kit}^{\text {high }}$ ILC2s are characterized by an "ILC3-like" phenotype with the expression of C-C chemokine receptor type 6 (CCR6) and the ability to produce IL-17A and IFN- $\gamma$ upon ILC3-stimulating cytokines. ${ }^{11}$ In contrast, c-Kit ${ }^{\text {low }}$ ILC2s represent fully mature ILC2s capable to produce type 2 cytokines. ${ }^{10}$

In vivo, ILC2s are stimulated by soluble mediators released by different cell types such as adipose cells, mast cells, epithelial cells ${ }^{12}$ and tumor cells. ${ }^{13}$ These activator signals comprise the interleukin (IL-) IL-25, the alarmin IL-33, ${ }^{14}$ the thymic stromal lymphopoietin (TSLP) and prostaglandins. ${ }^{15}$ In addition, ILC2 function can also be modulated by neuropeptides ${ }^{16}$ such as neuromedin U (NMU), ${ }^{17-20}$ vasoactive intestinal peptide (VIP) ${ }^{21}$ and calcitonin generelated peptide (CGRP). ${ }^{22-24}$ Upon activation, ILC2s are able to secrete type 2 cytokines such as IL-4, IL-5, IL-9 and $\mathrm{IL}-13,{ }^{25}$ highlighting their key role in the induction of type 2 responses.

Thus, several pathologies can be sustained by dysfunctional ILC2 $\mathrm{s}^{26}$ emphasizing the need to unravel the mechanisms underlying their chronic activation, to understand their role in type 2-mediated diseases. ${ }^{27,28}$ In this regard, the establishment of uniform tools to isolate and in vitro culture both human healthy donor- and patientderived ILC2s is needed to harmonize and compare datasets obtained by different research groups. ${ }^{29,30}$ Further, the comparison of the in vitro reactivity of ILC2s isolated from healthy donors (HDs) and patients remains poorly studied. It was previously reported that different culture conditions modulate human ILC2 functions and phenotype. Indeed, ILC2 cultured in Serum-Free Expansion Medium (SFEM II) exhibited superior survival compared to the ones expanded in Iscove Modified Dulbecco Medium (IMDM). However, when SFEM II was used in combination with IL-2, IL-25, IL-33 and TSLP, it induced minimal production of IL-5 and IL-13, while it sustained a higher production of IFN- $\gamma$, suggesting that SFEM II induced a phenotypic switch of ILC2s. ${ }^{31}$ In contrast, others reported that ILC2s expanded on the OP9 mouse stromal cells (OP9 cells) showed a limited plasticity. ${ }^{32}$

Here, we investigated the impact of culturing human ILC2s with IL-2 and IL-7, in combination or not with OP9 cells, a cocktail of cytokines composed of IL-25, IL-33 and TSLP and the mitogen phytohaemagglutinin (PHA). We assessed the effect of the different culture conditions on the proliferation, phenotype and function of ILC2s isolated from healthy donors or allergic patients.

Overall our results argue for major impacts of culture methods on ILC2 fitness, calling for careful selection of in vitro expansion methods in human ILC2 studies.

\section{Methods}

\section{Peripheral Blood Mononuclear Cell (PBMC) Isolation}

Venous blood from HDs ( $\mathrm{n}=6$ ) was collected at the local blood transfusion center, Lausanne, Switzerland, under the approval of the Lausanne University Hospital's Institute Review Board, upon written informed consent and in accordance with the Declaration of Helsinki. Allergic patient blood samples $(n=3)$ were obtained from the University Hospital of Geneva in the frame of the CCER 2017-00544 protocol, approved by the local Ethical Committee (Commission cantonale d'éthique de la recherche CCER, Rue Adrien-Lachenal 8, 1207 Geneva. The CCER is the overarching authority responsible for all studies in the canton of Geneva, including the ones performed at HUG. Dr Jandus is affiliated with HUG). Characteristics of patients are listed in Supplementary Table 1. PBMCs were freshly isolated by Lymphoprep (Promega) centrifugation (1800 rpm, 20 minutes, without break, room temperature). Red blood cell lysis was performed using red blood lysis buffer (Qiagen) and platelets were removed by centrifugation (1000 rpm, 10 minutes without break, room temperature). Cells were counted and immediately used.

\section{Flow Cytometry and ILC2 Isolation}

Human ILC2s were identified as lineage-negative lymphocytes. Lineage markers, all FITC conjugated included antihuman CD3 (UCHT1, Beckman Coulter BC), anti-human CD4 (SFCI12T4D11, BC), anti-human CD8 (MEM-31, Immunotools), anti-human CD14 (RMO52, BC), anti-human CD15 (80H5, BC), anti-human CD16 (3G8, BC), anti-human CD19 (J3-119, BC), anti-human CD20 (2H7, Biolegend), anti-human CD33 (HIM3-4, Biolegend), anti-human CD34 (561, Biolegend), anti-human CD94 (Miltenyi Biotech), antihuman 203c (E-NPP3 Biolegend), anti-human FceRI $\alpha$ (AER37, Biolegend). Additional markers used included Brilliant Violet 421 anti-human CD127 (A019D5, Biolegend), PerCPCy5.5 anti-human CRTH2 (BM16, Biolegend), Brilliant Violet 605 anti-human CD117 (c-Kit) (104D2, Biolegend), BUV737 anti-human CD56 (NCAM16.2, BD Biosciences), Brilliant Violet 785 anti-human CD94 (HP3D9, BD Biosciences), PE anti-human KLRG1 (13F12F2, 
eBiocsiences), PECy7 anti-human Tim3 (F38-2E2, eBiosciences), Brilliant Violet 711 anti-human PD-1 (EH12.2H7, Biolegend), Brilliant Violet 510 anti-human CD25 (BC96, Biolegend), Alexa700 anti-human CD137 (4B4-1, Biolegend), APC-Cy7 anti-human HLA-DR (I.243, Biolegend)and Brilliant Violet 650 anti-human CD69 (FN50, Biolegend). Samples were acquired on a LSRFortessa (BD) flow cytometer. Data analysis was performed using FlowJo software version 10.7.1. ILC2s were sorted to at least $98 \%$ purity using FACSAria (Becton Dickinson), based on the gating strategy illustrated in Figure 1A.

\section{Cell Culture}

Freshly sorted ILC2s were directly seeded after the FACSsorting in $200 \mu \mathrm{L}$ of RPMI-1640 (Eurobio) supplemented with $8 \%$ of human serum, $1 \%$ penicillin-streptomycin 10000U/mL (Gibco), 1\% L-Glutamine (Gibco), 1\% nonessential amino acids (Gibco), 1\% Na Pyruvate (Gibco), kanamycin $100 \mathrm{x}$ (Gibco) and $0.1 \%$ of $2 \beta$-mercaptoethanol $5 \times 10^{-2} \mathrm{M}$ (Sigma), supplemented with $100 \mathrm{U} / \mathrm{mL}$ recombinant human (rh) IL-2 (PeprtoTech) and 5ng/mL rhIL-7 (PeproTech). When indicated, 3000 mouse stromal OP9 cells (kindly provided by Prof. H. Spits and Dr B. Blum Amsterdam UMC Research Institutes), $1 \mu \mathrm{g} / \mathrm{mL}$ PHA (Sigma) or the cytokine cocktail composed of $50 \mathrm{ng} / \mathrm{mL}$ rhIL-25 (PeproTech), 50ng/mL rhIL-33 (PeproTech) and $50 \mathrm{ng} / \mathrm{mL}$ rhTSLP (PeproTech) were added. Cells were grown at $37^{\circ} \mathrm{C}$ under $5 \% \mathrm{CO}_{2}$.

\section{Cytokine Quantification}

The total ILC2s cultured in the different culture conditions were collected independently and washed with PBS. 2000 cells were re-seeded in 20U/mL rhIL-2 (PeproTech) and stimulated for 48 hours with 50ng/mL IL-25 (PeproTech), $50 \mathrm{ng} / \mathrm{mL}$ IL-33 (PeproTech) or a combination of both. Supernatant was collected and the concentration of various cytokines in ILC2 supernatants was determined using the bead-based immunoassay LEGENDplex ${ }^{\mathrm{TM}}$ analyte flow assay kit (human Th panel (13-plex), Biolegend) according to the manufacturer's protocol. Data were analyzed using Prism (GraphPad software version 8.4.2).

\section{Statistical Analysis}

Statistical analysis was performed using GraphPad Prism software version 8.4.2. One-way and two-way ANOVA tests were used. We compared all the conditions against the ex vivo level. For the between-condition comparisons, we compared "matched" conditions: conditions without and with the cytokine cocktail (i.e IL-2, IL-7 vs IL-2, IL$7+$ the cytokine cocktail; IL-2, IL-7 + OP9 vs IL-2, IL-7 + OP9 + cytokine cocktail; IL-2, IL-7 + PHA vs IL-2, IL-7 + PHA + cytokine cocktail). We also compared the three conditions without the cytokine cocktail and the three with the cytokine cocktail among each other (i.e IL-2, IL-7 vs IL-2, IL-7 + OP9 vs IL-2, IL-7 + PHA; IL-2, IL-7 + cytokine cocktail vs IL-2, IL-7 + cytokine cocktail + OP9 vs IL-2, IL-7 + cytokine cocktail + PHA). Statistically significant $\mathrm{p}$-value is represented with $*$. * = $<0.05, * *=<0.01, * * *=<0.001, * * * *=0.0001$

\section{Results}

\section{ILC2-Stimulating Cytokines Induced the} Highest in vitro Proliferation Rate and Favored c-Kit ${ }^{\text {low }}$ ILC2 Maintenance

To understand how different culture conditions can affect human ILC2 proliferation, phenotype and function, we freshly sorted total ILC2s from peripheral blood mononuclear cells (PBMCs) of HDs (Figure 1A) and expanded the cells as indicated in Figure 1B. Cells were put in culture with IL-2 and IL-7, supplemented either with OP9 cells or PHA. The same setting was used with the addition of a cytokine cocktail composed of IL-25, IL-33 and TSLP, known to activate ILC2s. ${ }^{7}$

As early as at day 7, we observed a difference in the proliferative capacity of ILC2s among the different conditions. In particular, the addition of the cytokine cocktail increased the proliferation of ILC2s independently from the presence of OP9 cells and/or PHA (Figure 1C). This initial observation was confirmed after 17-19 days of culture, when the presence of the cytokine cocktail fostered the highest proliferation (Figure 1D and E, Supp. Figure 1B). However, at this time point, we observed that the addition of OP9 cells strongly impaired the proliferative advantage given by the cytokine cocktail and that the addition of PHA did not further increase ILC2 proliferation. Taken together, these observations indicated that the cytokine cocktail but not the addition of stromal cells or a mitogen induced the highest proliferation of peripheral ILC2s in vitro.

Furthermore, we quantified and compared the proportion of c-Kit ${ }^{\text {high }}$ and c-Kit ${ }^{\text {low }}$ ILC2 populations ex vivo (Supp. Figure 1A) and after expansion (Figure $1 \mathrm{~F}$ and G). The distribution of c-Kit ${ }^{\text {high }}$ and c-Kit ${ }^{\text {low }}$ ILC2s was dramatically modulated by the culture conditions. Indeed, a switch in the repartition of both populations occurred with the c-Kit ${ }^{\text {high }}$ ILC2s increasing when the cells were cultured with IL-2, IL- 
A
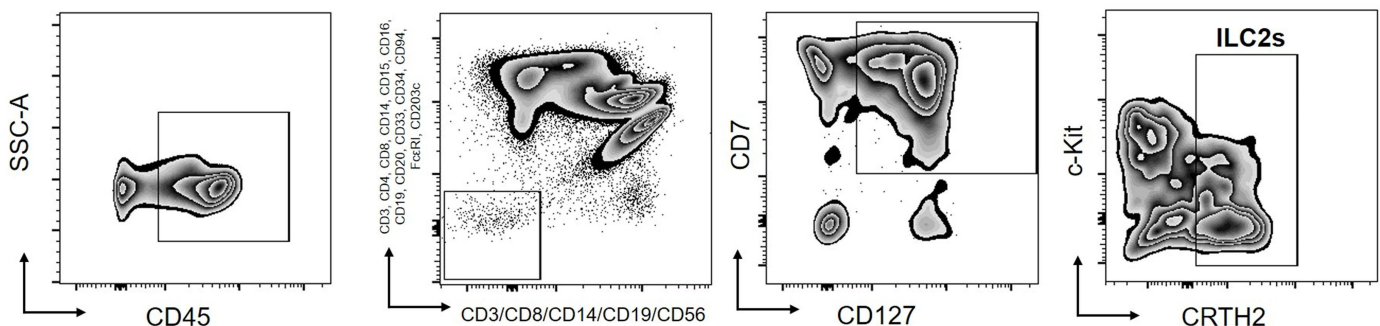

B

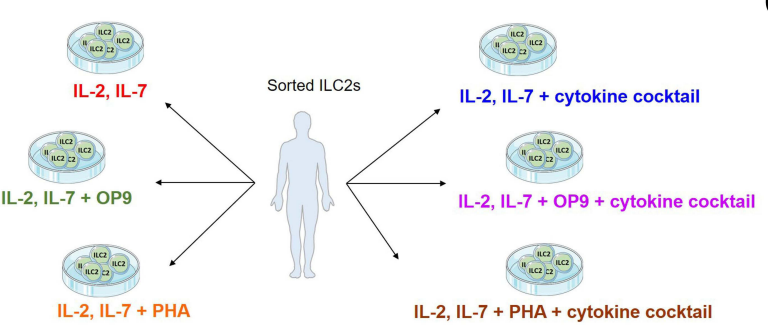

C
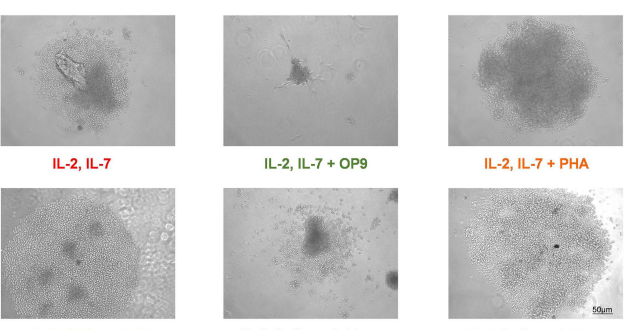

IL-2, IL-7 + cytokine

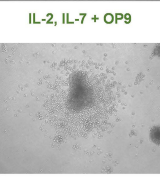

IL-2, IL-7 + PHA

E
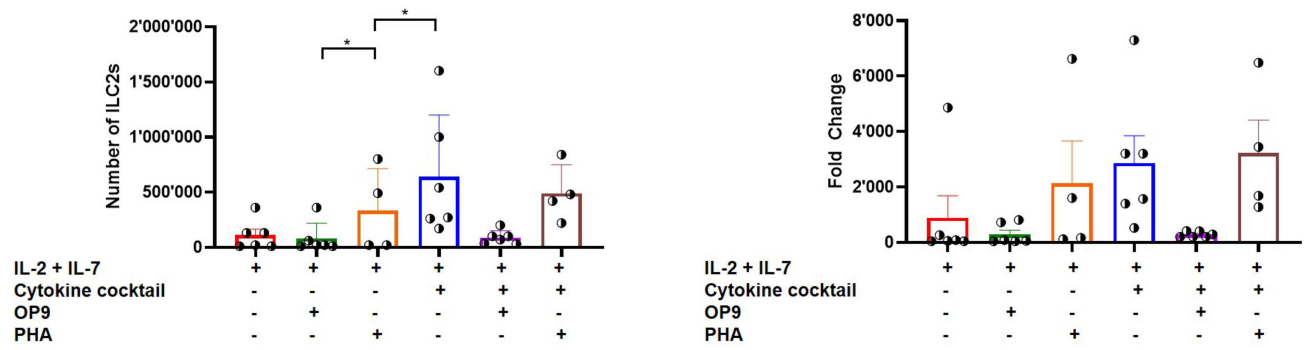

F

Ex vivo

IL-2, IL-7

IL-2, IL-7
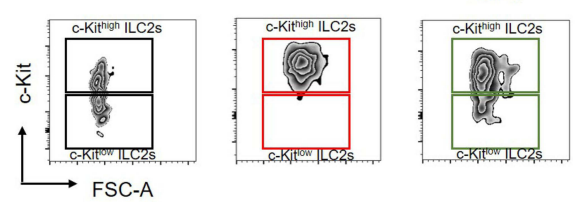

IL-2, IL-7
+ PHA

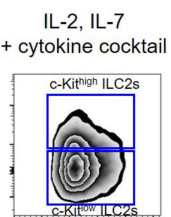

IL-2, IL-7

IL-2, IL-7

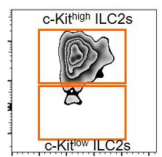

OP9

$+\mathrm{PHA}$
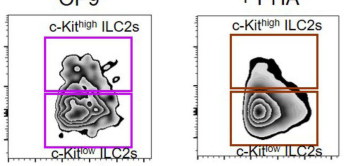

G
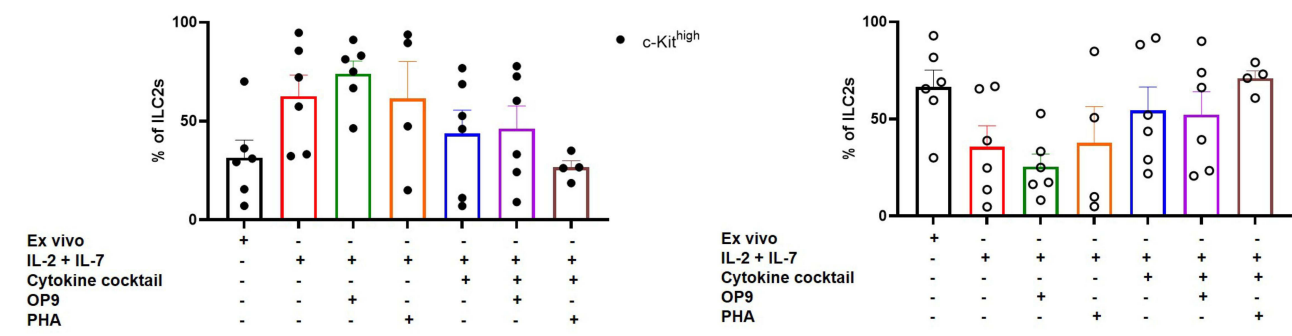

Figure I ILC2-stimulating cytokines induced the highest in vitro proliferation rate and favored c-Kit ${ }^{\text {low }}$ cell maintenance. (A) Gating strategy used to sort fresh ILC2s from PBMCs. (B) Scheme representing the different culture conditions used in the experiment. Freshly sorted ILC2s were put in culture in medium with IL2 and IL-7, supplemented either with mouse stromal OP9 cells ( $n=6 \mathrm{HD}$ samples from 4 independent experiments) or PHA ( $\mathrm{n}=4 \mathrm{HD}$ samples from 4 independent experiments). The same setting was used with the addition of a cytokine cocktail composed of IL-25, IL-33 and TSLP ( $\mathrm{n}=$ between 4 and 6 HD samples from 4 independent experiments). (C) Representative examples of expanded ILC2s in the different culture conditions ( $n=$ between 4 and 6 HD samples from 4 independent experiments) (magnification $=10 x$ ). (D) Quantification of the ILC2s counted after 17-19 days of expansion for each culture condition ( $n=4 / 6$ ). (E) Fold change of ILC2s after the culture relative to initially plated cells. (F) Representative dotplots of c-Kit ${ }^{\text {high }}$ and c-Kit ${ }^{\text {low }}$ ILC2 populations ex vivo and after expansion in each culture condition ( $\mathrm{n}=$ between 4 and $6 \mathrm{HD}$ samples from 4 independent experiments). (G) Quantification of c-Kit ${ }^{\text {high }}$ and $\mathrm{c}-\mathrm{Kit}{ }^{\text {low }}$ ILC2 populations ex vivo and after expansion in each culture condition ( $n=$ between 4 and 6 HD samples from 4 independent experiments). Data are shown as mean \pm standard error of the mean (SEM). Significance was calculated using one-way ANOVA where $* \mathrm{P}<0.05$. 
7, with or without OP9 cells and/or PHA (Figure 1G). In contrast, the expansion of ILC2s with the cytokine cocktail, irrespective of the presence of OP9 cells and/or PHA, favored the c-Kit ${ }^{\text {low }}$ ILC2s. Interestingly, ILC2s cultured with the cytokine cocktail and PHA revealed the most homogenous trend across donors, with a majority of c-Kit ${ }^{\text {low }}$ ILC2s (Figure 1G), suggesting the maintenance of a bona fide mature ILC2 state.

\section{Different Culture Conditions Affected Marker Expression in $\mathrm{c}-\mathrm{Kit}^{\text {high }}$ and c-Kit ${ }^{\text {low }}$ ILC2s}

In order to decipher the phenotype of both $\mathrm{c}-\mathrm{Kit}^{\text {high }}$ and c-Kit ${ }^{\text {low }}$ ILC2s ex vivo and among the different culture conditions, we assessed the expression and the modulation of a selection of markers involved in ILC2 biology. We first assessed the expression of CD25, known to be expressed by human ILC2s under physiological conditions in some tissues ${ }^{33}$ and by cytokine activated ILC2s in vitro. ${ }^{34} \mathrm{CD} 25$ expression was not modulated and remained high among all the conditions in both $\mathrm{c}-\mathrm{Kit}^{\text {high }}$ and $\mathrm{c}-\mathrm{Kit}{ }^{\text {low }}$ ILC2s (Figure 2A). We then evaluated the expression of another ILC2-specific marker, Killer cell Lectin-like Receptor subfamily G member 1 (KLRG1), a co-inhibitory receptor. ${ }^{35}$ The expression of KLRG1 tended to decrease in culture compared to ex vivo. Notably, the cytokine cocktail in the presence of OP9 cells induced the strongest reduction of KLRG1 expression (Figure 2B). Programmed Cell Death 1 (PD-1) has

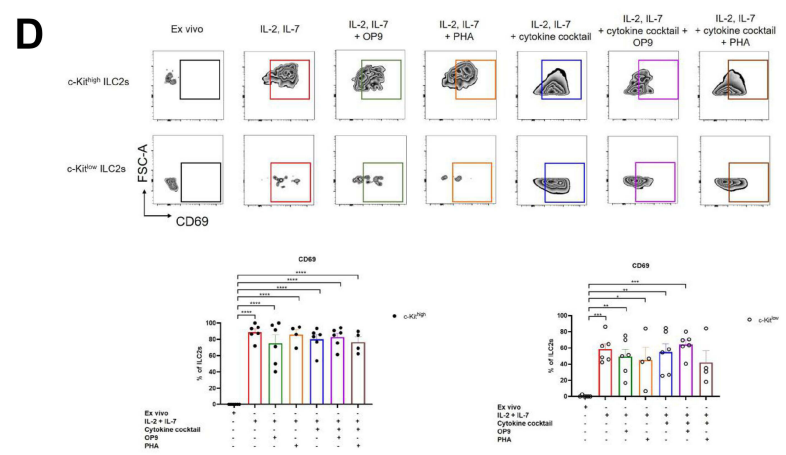

$\mathbf{E}$

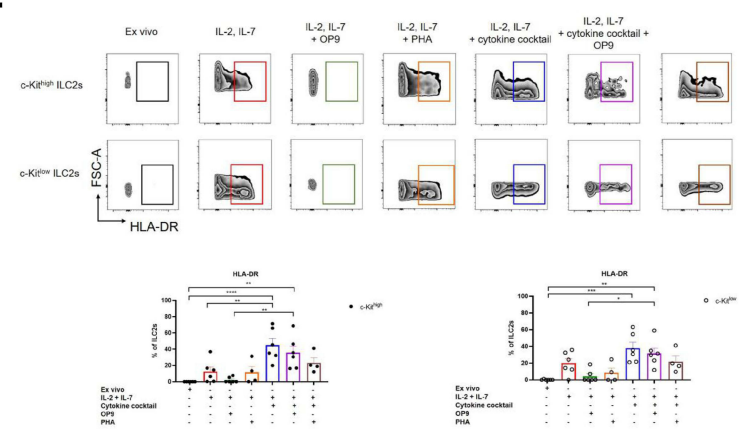

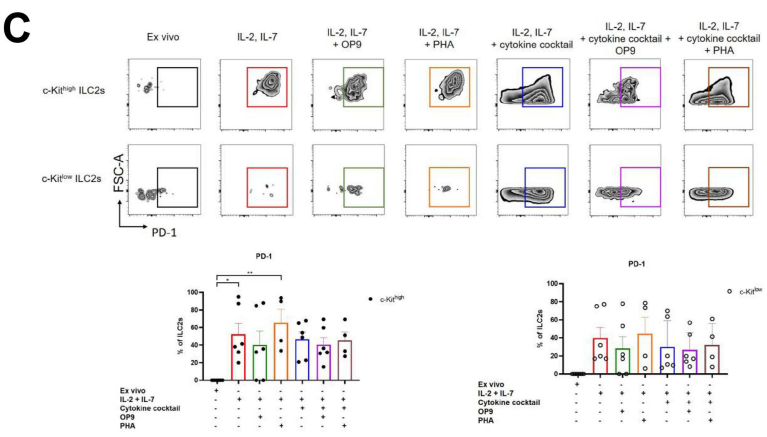

Figure 2 Different culture conditions affected marker expression in c-Kit ${ }^{\text {high }}$ and c-Kit ${ }^{\text {low }}$ ILC2s. Representative dotplots and quantification of CD25 (A), KLRG I (B), PD- I (C), CD69 (D) and HLA-DR (E) in ex vivo and in vitro expanded ILC2s, across different culture conditions ( $\mathrm{n}=$ between 4 and $6 \mathrm{HD}$ samples from 4 independent experiments). Data are shown as mean \pm standard error of the mean (SEM). Significance was calculated using one-way ANOVA where $* \mathrm{P}<0.05$, $* * \mathrm{P}<0.0 \mathrm{I}$, $* * * \mathrm{P}<0.00 \mathrm{I}$, $* * * * \mathrm{P}<0.0001$. 
been identified as regulator of $\mathrm{KLRG}^{+} \mathrm{ILC} 2$ function in humans. $^{36,37}$ As shown in Figure $2 \mathrm{C}$, we did not detect PD-1 expression on ex vivo ILC2s, whereas the culture induced its expression, which was significantly higher in

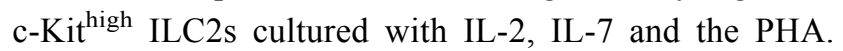
Next, we assessed the expression of the lymphocyte activation marker CD69, ${ }^{38}$ known to be expressed by activated ILC2s. ${ }^{39}$ Ex vivo ILC2s did not express CD69 in contrast to expanded ILC2s that expressed it at high level, irrespective of the culture conditions (Figure 2D). In addition, the MHC class II molecule human leukocyte antigen-D related (HLA-DR) known to modulate the antigen presenting capacity of ILC $2 \mathrm{~s}^{40}$ and more recently identified as being regulated by cytokines $^{41}$ was monitored. HLA-DR was not detected on ex vivo ILC2s, in line with the literature. ${ }^{33,41}$ However, HLA-DR expression increased in both c-Kithigh and c-Kit ${ }^{\text {low }}$ ILC2s expanded with the cytokine cocktail, with or without OP9 cells (Figure 2E). Last, we also evaluated the expression of the inhibitory receptor T-cell immunoglobulin and mucin-domain containing-3 $(\operatorname{Tim} 3)^{42}$ and the co-stimulatory molecule $\mathrm{CD} 137^{43}$ which were however not detectable (Supp. Figure 2A and $\mathrm{B}$ ).

\section{ILC2s Expanded with IL-2 and IL-7 and Re-Challenged with IL-33 Displayed an Increased IL-5 and IL- I 3 Secretion}

ILC2 function is mainly associated with the production of type 2 cytokines, such as IL-4, IL-5, IL-9 and IL-13. ${ }^{44}$ Thus, to investigate whether the different culture conditions affected ILC2 function, we evaluated the cytokine secretion profile of total ILC2s after culture. Cells were harvested, washed and re-seeded to be stimulated for 48 hours with IL-2 as control, with IL-25 and IL-33 alone or in combination (Figure 3A). At the end of the stimulation, the secreted cytokines were quantified in the supernatants.

Upon IL-33 stimulation alone or in combination with IL25 , only the ILC2s expanded with IL-2 and IL-7 showed an increased production of both IL-5 (Figure 3B) and IL-13 (Figure 3D). This effect was independent from the addition of OP9 cells or PHA during the expansion phase (Figure 3B and D). Notably, the ILC2s expanded with the cytokine cocktail showed a high and similar level of IL-5 and IL-13 among the different re-challenge conditions (Figure $3 \mathrm{C}$ and $\mathrm{E}$ ). Further, IL-25 alone did not induce neither IL-5 nor IL-13 secretion compared to IL-2 stimulation, suggesting that the higher secretion observed with the combination of IL-25 and IL-33 was mainly driven by IL-33.

In addition, we also evaluated the secretion of IL-4 and IL-9, other two well-known ILC2-secreted cytokines. ${ }^{45,46}$ We did not observe any significant modulation among the different culture conditions and stimulations (Supp. Figure 3A-D). However, IL-9 tended to be more elevated in cultures of ILC2 expanded with IL-2 and IL-7 upon IL33 re-stimulation (Supp. Figure 3A), while IL-4 was lower in ILC2s expanded with the cytokine cocktail and OP9 cells (Supp. Figure 3C).

High plasticity is a key feature of human ILC $2 s^{47,48}$ that can be driven by cytokine exposure. ${ }^{49,50}$ Consequently, to decipher whether the different culture conditions induced ILC2 plasticity and thus a switch towards another type of cytokine-secreting profile, we examined the secretion of ILC1 and ILC3 prototypic cytokines, IFN- $\gamma$, IL-17A and IL-17F, respectively. None of the culture conditions induced a secretion of these cytokines (Supp. Figure 3E-J). Nevertheless, we noticed a trend of increased IFN- $\gamma$ secretion by some ILC2s expanded with the cytokine cocktail and re-challenged with IL-25 and IL-33 (Supp. Figure 3F).

\section{Distinct in vitro Behavior of Healthy Donor-Derived and Patient ILC2s}

ILC2s have been associated with various allergic disorders. ${ }^{5}$ For instance, patients suffering from allergic asthma displayed an enhanced type 2 immunity in peripheral blood and in lungs, ${ }^{51,52}$ and an ILC2 decrease was observed in patients responding to allergen-specific immunotherapy (AIT), but not in non-responders. ${ }^{53}$ To evaluate whether ILC2s from allergic patients showed the same in vitro behavior as healthy ILC2s, we performed the same experiments described above using freshly isolated ILC2s from allergic patients' PBMCs and we made a side by side comparison between HD and patient cells.

First of all, we observed that the frequency of ex vivo total ILC2s was similar between HDs and patients but with an opposite repartition in the frequency of $\mathrm{c}-\mathrm{Kit}^{\text {high }}$ and c-Kit ${ }^{\text {low }}$, with expanded c-Kit ${ }^{\text {high }}$ ILC2s in patients (Figure 4A, Supp. Figure 4E). In vitro, we observed that the subsets repartition was differently modulated in HD and patient ILC2s (Figure 4A). In patient but not in HDs, the expansion with IL-2 and IL-7 favored the maintenance of $\mathrm{c}-\mathrm{Kit}^{\text {high }}$ ILC2s (Supp. Figure 4D and $\underline{\mathrm{E}}$, Figure 1G, Figure 4A). Moreover, the addition of the 


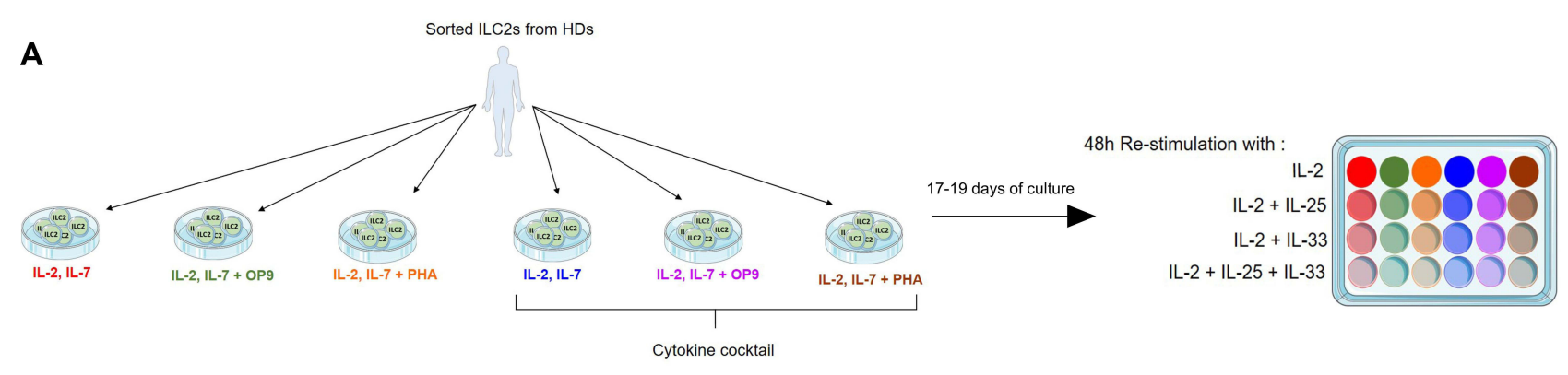

B B
L-2.
LL-25
LL-33

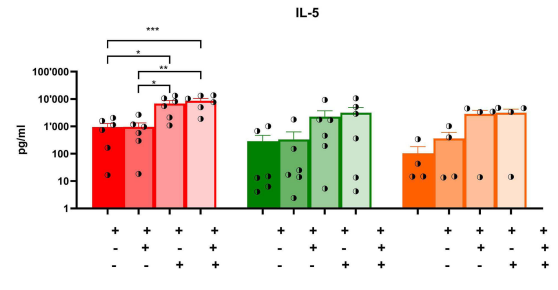

D
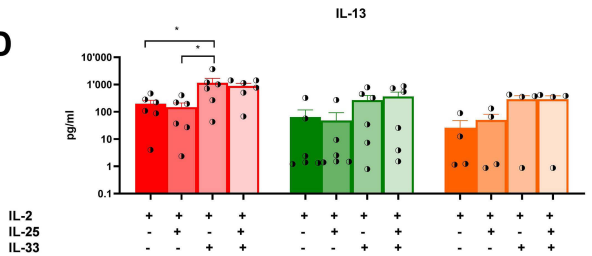

- IL-2, LL-7

IL-2, IL-7 + OP9
IL-2, IL-7 + PHA

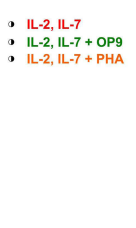

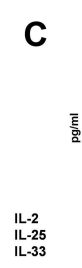
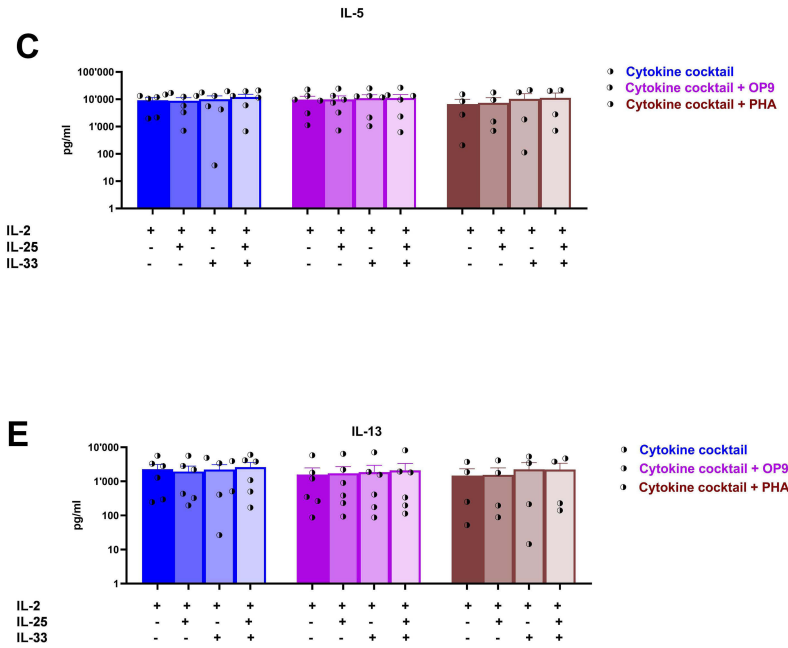

- Cytokine cocktail

Cytokine cocktail + OPg

Figure 3 ILC2s expanded with IL-2 and IL-7 and re-challenged with IL-33 displayed an increased IL-5 and IL- I 3 secretion. (A) Schematic representation of the experimental design: after the expansion, ILC2s from each condition ( $n=$ between 4 and 6 HD samples from 4 independent experiments) were separately harvested and re-plated for a 48 hours cytokine stimulation. Cells were stimulated with IL-2 as control, added with IL-25, IL-33 or a combination of both. The readout was performed using the LEGENDplex assay kit. ILC2s expanded with IL-2 and IL-7 ( $\mathrm{n}=6 \mathrm{HD}$ samples from 4 independent experiments) secreted more IL-5 (B) and IL-I3 (C) upon IL-33 stimulation. IL-5 (D) and IL-I3 (E) secretion were not modulated upon the different stimulations when ILC2s were expanded with the cytokine cocktail, added or not with OP9 cells ( $=6$ HD samples from 4 independent experiments) or PHA ( $n=4$ HD samples from 4 independent experiments). Data are shown as mean \pm standard error of the mean (SEM). Significance was calculated using one-way ANOVA where $* \mathrm{P}<0.05, * * \mathrm{P}<0.01$, $* * * \mathrm{P}<0.00$ I.

cytokine cocktail in the expansion phase favored the c-kit $^{\text {low }}$ subset of patient ILC2s (Figure 4A, Supp. Figure 4E), while in HDs, c-Kit ${ }^{\text {low }}$ ILC2s were maintained by the expansion with the cytokine cocktail and PHA (Figure 1G). In addition, by analyzing the proliferation of the cells, we observed that patient $\mathrm{c}-\mathrm{Kit}^{\text {high }}$ and c-Kit ${ }^{\text {low }}$ ILC2s displayed a higher proliferation when expanded with PHA (Supp. Figure 4A-C, Supp. Figure $4 \mathrm{~F}$ ), in contrast to HD ILC2s that proliferated more when expanded with the cytokine cocktail (Figure 1D-E). Next, by comparing HD and patient ILC2 phenotype, we observed that the expansion of patient ILC2s with OP9 cells downregulated the expression of activation markers such as PD-1, KLRG1, CD69 and HLA-DR (Figure 4B, Supp. Figure 5B-E). Nonetheless, the expression of $\mathrm{CD} 25$ remained similar in both HD and patient ILC2s (Figure 4B, Supp. Figure 5A), as well as the lack of expression of Tim3 and CD137 (Supp. Figure 5F-G).

Finally, by assessing the IL-5 and IL-13 secretion profile between HD and patient ILC2s, we observed an overall lower level of secretion in patients (Figure 4C). Furthermore, the presence of OP9 cells during the expansion tended to impair IL-5 and IL-13 production upon re-stimulation of patient ILC2s (Figure 4C, Supp. Figure 6A-D). No other type-2, type-1 or -3 cytokines were detected in patient ILC2 cultures, irrespective of the condition (Supp. Figure $6 \mathrm{E}-\underline{\mathrm{N}}$ ).

\section{Discussion}

Recently, a rapidly growing body of literature has integrated the ILC2 profiling in the immune monitoring portfolio of several type 2-mediated disease settings. The 
A

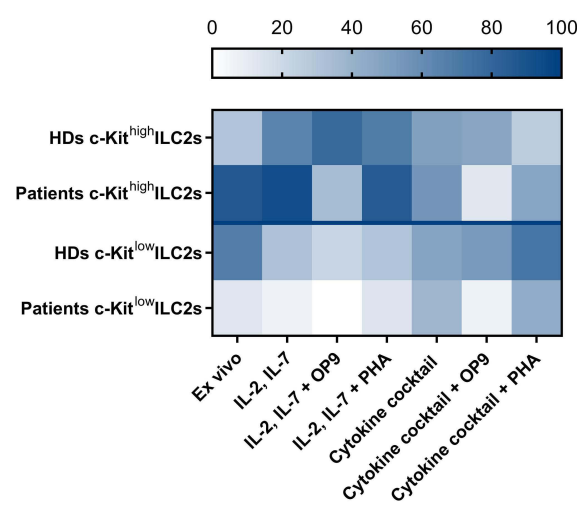

C
IL-5
IL-13

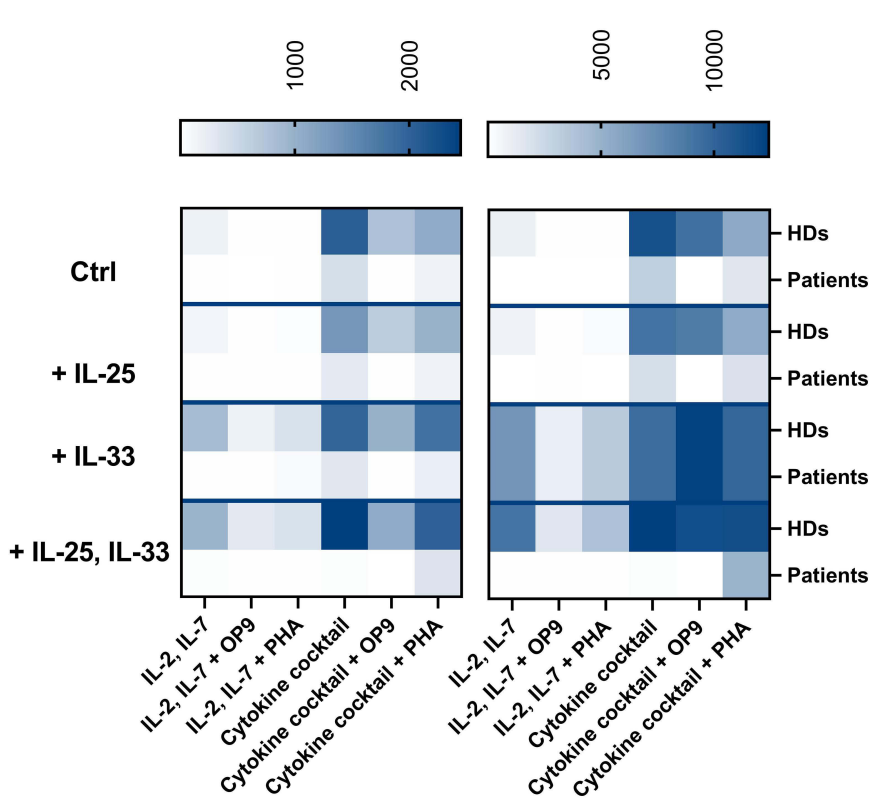

B

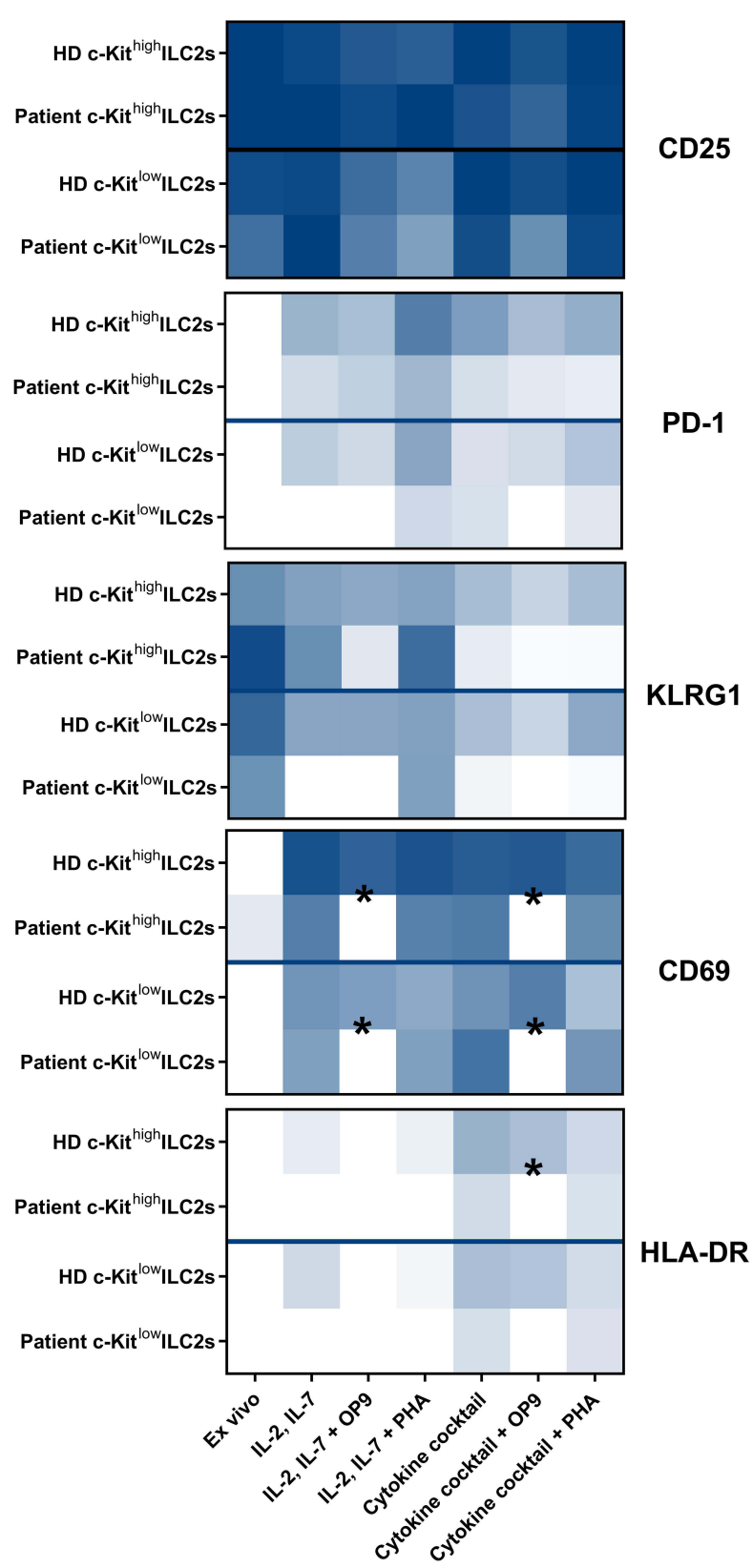

Figure 4 Distinct in vitro behavior of healthy donor-derived and patients ILC2s. Heat maps representing the comparison of HDs ( $n=$ between 4 and 6 HD samples from 4 independent experiments) and allergic patients ( $n=3$ allergic patient samples from 2 independent experiments) for the proportion of c-Kit ${ }^{\text {high }}$ and c-Kit ${ }^{\text {low }}$ ILC2s ex vivo and after the expansion in the different culture conditions (A), the activation marker expression (B) and the cytokine secretion profile upon re-stimulation (C). Data are shown as mean \pm standard error of the mean (SEM). Significance was calculated using two-way ANOVA where $* P<0.05$.

analysis of these cells ex vivo relies on more and more standardized multiparametric flow cytometry panels that provide knowledge on ILC2 phenotype and frequency. Instead, their in-depth functional characterization mostly depends on in vitro studies or on the use of genetically engineered mouse models. ${ }^{40}$ Here, we provide an extensive comparison of the impact of in vitro culture conditions on human ILC2 fitness and phenotype, arguing for the need of more harmonized protocols for in vitro ILC2 functional studies.

It has previously been shown that the expansion of ILC2s in SFEM II media induced a plastic phenotype 
with cells that gained the ability to produce IFN- $\gamma$ and IL$17 \mathrm{~A}$, in the presence or not of IL-25, IL-33 and TSLP. ${ }^{31}$ We did not observe any switch towards IFN- $\gamma$ and IL-17A/ IL-17F secretion using RPMI medium supplemented with $8 \%$ human serum (HS), even when ILC2s were expanded with the cytokine cocktail and re-challenged with either IL-25 or IL-33 or both. However, one can speculate that depending on the use of FCS or HS, and on the glucose and/or amino acid content of the medium, ILC2 biology/ plasticity will be dramatically affected. This is mainly due to the known distinct metabolic requirements of ILC subsets, being resting ILC2 primarily fatty-acid ${ }^{54,55}$ and amino-acid ${ }^{56}$ dependent, at least in the intestine and the lung, respectively. The rationale to include HS was to mimic as close as possible the ex-vivo conditions of human peripheral blood circulating cells.

Further, ILC2-driver cytokines have been increasingly characterized in the last years. ${ }^{57}$ Here, we demonstrated that the addition of these mediators (eg IL-33, IL-25) during the in vitro expansion phase leads to the highest cell proliferation rate. Yet, this pre-exposure seems to shape ILC2s, since upon short-term re-challenge with different cytokines to monitor their functionality, a similar type- 2 cytokine plateau is observed across conditions. Therefore, while cytokine-driven expansion is suitable to obtain large cell numbers, it seems counterproductive if measurement of cell functionality is the primary study aim. In that regard, it would be interesting to compare the reactivity of ILC2s isolated from allergic patients under immunotherapy. Indeed, based on the induction of "regulatory" IL-10-secreting ILC2s upon AIT in allergic patients, one can hypothesize that differences in functional polarization (eg IL-10 versus IL-13 secretion) might still be captured when cytokines are used during the in vitro cell propagation. ${ }^{58}$ Moreover, it would be informative to test whether a resting period in IL-2 and IL-7 only after expansion in the presence of IL-25 and IL-33 would restore their capacity to respond to a further in vitro stimulation.

A further aspect related to ILC expansion is linked to the use of stromal cells to support their growth. The addition of OP9 cells or Notch ligand Delta-like 4 expressing OP9 (OP9-DL4) as feeders has been described to support human ILC2 clonal expansion. ${ }^{32,49}$ OP9-DL1 expressing the Notch ligand Delta-like 1 has also been identified as promoting ILC2 differentiation. ${ }^{35}$ However, in our hands, the addition of OP9 cells had a detrimental effect on ILC2 proliferation, in both allergic patient and healthy donor
ILC2s. Furthermore, we observed that OP9 cells impaired patient ILC2 functions, since their type- 2 cytokine secretion was dramatically reduced. One can speculate that OP9-secreted factors ${ }^{59}$ might directly affect cytokine secretion. Nevertheless, in line with previous observations, ${ }^{49}$ we also observed that the addition of OP9 cells restrains ILC2 plasticity. Further, in healthy ILC2s, our data indicate that the addition of OP9 cells with IL-2 and IL-7 favored the maintenance of the c-Kithigh subset, while the combination of the cytokine cocktail and PHA supported a fully activated state of ILC2s as observed with a high proportion of $\mathrm{c}-\mathrm{Kit}^{\text {low }}$ ILC2s after expansion.

To conclude, we demonstrated that the proliferation behavior and the functions of ILC2s can be distinctly modulated, depending on the conditions used for their in vitro expansion. Moreover, we also showed that ILC2s isolated from HDs and allergic patients are differently affected by the in vitro conditions used for their expansion. Overall, we believe that the development of refined in vitro culture system for ILC2 will be of invaluable use to profile and exploit these cells in human type- 2 mediated disorders.

\section{Ethics Approval and Informed Consent}

Venous blood was collected at the local Blood transfusion center, upon written informed consent and in accordance with the Declaration of Helsinki. Allergic patient blood samples were obtained from the University Hospital of Geneva (HUG) in the frame of the CCER 2017-00544 protocol, approved by the local Ethical Committee (Commission cantonale d'éthique de la recherche CCER, Rue Adrien-Lachenal 8, 1207 Geneva) and upon written informed consent. The CCER is the overarching authority responsible for all studies in the canton of Geneva, including the ones performed at HUG. Dr Jandus is affiliated with HUG.

\section{Acknowledgments}

We thank healthy donors and patients for the participation to this study. This work was supported by grant from the Swiss National Science Foundation (PRIMA_PR00P3_179727) to $\mathrm{CJ}$ and Fondazione Umberto Veronesi to GE.

Data available on request from the authors https://doi. org/10.26037/yareta:kjxpl2iuwjhprnv3gudbuoasdm. 


\section{Author Contributions}

All authors made a significant contribution to the work reported, whether that is in the conception, study design, execution, acquisition of data, analysis and interpretation, or in all these areas; took part in drafting, revising or critically reviewing the article; gave final approval of the version to be published; have agreed on the journal to which the article has been submitted; and agree to be accountable for all aspects of the work.

\section{Disclosure}

The authors declare no conflicts of interest in this work.

\section{References}

1. Galli SJ, Tsai M, Piliponsky AM. The development of allergic inflammation. Nature. 2008;454(7203):445-454. doi:10.1038/ nature 07204

2. Eberl G, Colonna M, Di Santo JP, et al. Innate lymphoid cells. Innate lymphoid cells: a new paradigm in immunology. Science. 2015;348 (6237):aaa6566. doi:10.1126/science.aaa6566

3. Licona-Limon P, Kim LK, Palm NW, et al. TH2, allergy and group 2 innate lymphoid cells. Nat Immunol. 2013;14(6):536-542. doi:10.1038/ni.2617

4. McKenzie AN. Type-2 innate lymphoid cells in asthma and allergy. Ann Am Thorac Soc. 2014;11(Suppl 5):S263-70. doi:10.1513/ AnnalsATS.201403-097AW

5. Pasha MA, Patel G, Hopp R, et al. Role of innate lymphoid cells in allergic diseases. Allergy Asthma Proc. 2019;40(3):138-145. doi:10.2500/aap.2019.40.4217

6. Doherty TA, Broide DH. Airway innate lymphoid cells in the induction and regulation of allergy. Allergol Int. 2019;68(1):9-16. doi:10.1016/j.alit.2018.11.001

7. Vivier E, Artis D, Colonna M, et al. Innate lymphoid cells: 10 years on. Cell. 2018;174(5):1054-1066. doi:10.1016/j.cell.2018.07.017

8. Wojno ED, Monticelli LA, Tran SV, et al. The prostaglandin D(2) receptor CRTH2 regulates accumulation of group 2 innate lymphoid cells in the inflamed lung. Mucosal Immunol. 2015;8(6):1313-1323. doi:10.1038/mi.2015.21

9. Mjosberg JM, Trifari S, Crellin NK, et al. Human IL-25- and IL-33responsive type 2 innate lymphoid cells are defined by expression of CRTH2 and CD161. Nat Immunol. 2011;12(11):1055-1062. doi:10.1038/ni.2104

10. Hochdorfer T, Winkler C, Pardali K, et al. Expression of c-Kit discriminates between two functionally distinct subsets of human type 2 innate lymphoid cells. Eur J Immunol. 2019;49(6):884-893. doi:10.1002/eji.201848006

11. Bernink JH, Ohne Y, Teunissen MBM, et al. c-Kit-positive ILC2s exhibit an ILC3-like signature that may contribute to IL-17-mediated pathologies. Nat Immunol. 2019;20(8):992-1003. doi:10.1038/ s41590-019-0423-0

12. Brestoff JR, Kim BS, Saenz SA, et al. Group 2 innate lymphoid cells promote beiging of white adipose tissue and limit obesity. Nature. 2015;519(7542):242-246. doi:10.1038/nature14115

13. Trabanelli S, Chevalier MF, Martinez-Usatorre A, et al. Tumourderived PGD2 and NKp30-B7H6 engagement drives an immunosuppressive ILC2-MDSC axis. Nat Commun. 2017;8(1):593. doi:10.1038/s41467-017-00678-2

14. Neill DR, Wong SH, Bellosi A, et al. Nuocytes represent a new innate effector leukocyte that mediates type-2 immunity. Nature. 2010;464(7293):1367-1370. doi:10.1038/nature08900
15. Maric J, Ravindran A, Mazzurana L, et al. Cytokine-induced endogenous production of prostaglandin D2 is essential for human group 2 innate lymphoid cell activation. J Allergy Clin Immunol. 2019;143 (6):2202-2214 e5. doi:10.1016/j.jaci.2018.10.069

16. Klose CS, Artis D. Neuronal regulation of innate lymphoid cells. Curr Opin Immunol. 2019;56:94-99. doi:10.1016/j.coi.2018.11.002

17. Wallrapp A, Riesenfeld SJ, Burkett PR, et al. The neuropeptide NMU amplifies ILC2-driven allergic lung inflammation. Nature. 2017;549 (7672):351-356. doi:10.1038/nature24029

18. Sui P, Wiesner DL, Xu J, et al. Pulmonary neuroendocrine cells amplify allergic asthma responses. Science. 2018;360(6393):6393. doi: $10.1126 /$ science.aan 8546

19. Cardoso V, Chesné J, Ribeiro H, et al. Neuronal regulation of type 2 innate lymphoid cells via neuromedin U. Nature. 2017;549 (7671):277-281. doi:10.1038/nature23469

20. Klose CSN, Mahlakõiv T, Moeller JB, et al. The neuropeptide neuromedin $\mathrm{U}$ stimulates innate lymphoid cells and type 2 inflammation. Nature. 2017;549(7671):282-286. doi:10.1038/nature23676

21. Nussbaum JC, Van Dyken SJ, von Moltke J, et al. Type 2 innate lymphoid cells control eosinophil homeostasis. Nature. 2013;502 (7470):245-248. doi:10.1038/nature12526

22. Nagashima H, Mahlakõiv T, Shih H-Y, et al. Neuropeptide CGRP limits group 2 innate lymphoid cell responses and constrains type 2 inflammation. Immunity. 2019;51(4):682-695. doi:10.1016/j. immuni.2019.06.009

23. Wallrapp A, Burkett PR, Riesenfeld SJ, et al. Calcitonin gene-related peptide negatively regulates alarmin-driven type 2 innate lymphoid cell responses. Immunity. 2019;51(4):709-723 e6. doi:10.1016/j. immuni.2019.09.005

24. Xu H, Ding J, Porter CBM, et al. Transcriptional atlas of intestinal immune cells reveals that neuropeptide alpha-CGRP modulates group 2 innate lymphoid cell responses. Immunity. 2019;51(4):696-708 e9. doi:10.1016/j.immuni.2019.09.004

25. Serafini N, Vosshenrich CA, Di Santo JP. Transcriptional regulation of innate lymphoid cell fate. Nat Rev Immunol. 2015;15(7):415-428. doi: $10.1038 / \mathrm{nri3} 355$

26. Sciume G, Fionda C, Stabile H, et al. Negative regulation of innate lymphoid cell responses in inflammation and cancer. Immunol Lett. 2019;215(p):28-34. doi:10.1016/j.imlet.2019.01.011

27. Kabata H, Moro K, Koyasu S. The group 2 innate lymphoid cell (ILC2) regulatory network and its underlying mechanisms. Immunol Rev. 2018;286(1):37-52. doi:10.1111/imr.12706

28. Ducimetiere L, Vermeer M, Tugues S. The interplay between innate lymphoid cells and the tumor microenvironment. Front Immunol. 2019;10(p):2895. doi:10.3389/fimmu.2019.02895

29. Trabanelli S, Gomez-Cadena A, Salomé B, et al. Human innate lymphoid cells (ILCs): toward a uniform immune-phenotyping. Cytometry B Clin Cytom. 2018;94(3):392-399. doi:10.1002/cyto.b.21614

30. Spits H, Artis D, Colonna M, et al. Innate lymphoid cells--a proposal for uniform nomenclature. Nat Rev Immunol. 2013;13(2):145-149. doi: $10.1038 /$ nri3365

31. Camelo A, Rosignoli G, Ohne Y, et al. IL-33, IL-25, and TSLP induce a distinct phenotypic and activation profile in human type 2 innate lymphoid cells. Blood Adv. 2017;1(10):577-589. doi:10.1182/ bloodadvances. 2016002352

32. Lim AI, Li Y, Lopez-Lastra S, et al. Systemic human ILC precursors provide a substrate for tissue ILC differentiation. Cell. 2017;168 (6):086-1100 e10. doi:10.1016/j.cell.2017.02.021

33. Simoni Y, Fehlings M, Kløverpris HN, et al. Human innate lymphoid cell subsets possess tissue-type based heterogeneity in phenotype and frequency. Immunity. 2017;46(1):148-161. doi:10.1016/j. immuni.2016.11.005

34. Maric J, Ravindran A, Mazzurana L, et al. Prostaglandin E2 suppresses human group 2 innate lymphoid cell function. J Allergy Clin Immunol. 2018;141(5):1761-1773. doi:10.1016/j.jaci.2017.09.050 
35. Nagasawa M, Heesters BA, Kradolfer CMA, et al. KLRG1 and NKp46 discriminate subpopulations of human CD117(+)CRTH2(-) ILCs biased toward ILC2 or ILC3. J Exp Med. 2019;216 (8):1762-1776. doi:10.1084/jem.20190490

36. Taylor S, Huang Y, Mallett G, et al. PD-1 regulates KLRG1(+) group 2 innate lymphoid cells. J Exp Med. 2017;214(6):1663-1678. doi:10.1084/jem.20161653

37. Chiossone L, Vivier E. Immune checkpoints on innate lymphoid cells. $J$ Exp Med. 2017;214(6):1561-1563. doi:10.1084/ jem.20170763

38. Shiow LR, Rosen DB, Brdičková N, et al. CD69 acts downstream of interferon-alpha/beta to inhibit S1P1 and lymphocyte egress from lymphoid organs. Nature. 2006;440(7083):540-544. doi:10.1038/ nature 04606

39. Munneke JM, Björklund AT, Mjösberg JM, et al. Activated innate lymphoid cells are associated with a reduced susceptibility to graft-versus-host disease. Blood. 2014;124(5):812-821. doi:10.1182/ blood-2013-11-536888

40. Oliphant CJ, Hwang Y, Walker J, et al. MHCII-mediated dialog between group 2 innate lymphoid cells and $\mathrm{CD} 4(+) \mathrm{T}$ cells potentiates type 2 immunity and promotes parasitic helminth expulsion. Immunity. 2014;41(2):283-295. doi:10.1016/j.immuni.2014.06.016

41. Rao A, Strauss O, Kokkinou E, et al. Cytokines regulate the antigen-presenting characteristics of human circulating and tissue-resident intestinal ILCs. Nat Commun. 2020;11(1):2049. doi:10.1038/s41467-020-15695-x

42. He Y, Cao J, Zhao C, et al. TIM-3, a promising target for cancer immunotherapy. Onco Targets Ther. 2018;11:7005-7009. doi:10.2147/OTT.S170385

43. Chu DT, Bac ND, Nguyen K-H, et al. An update on anti-CD137 antibodies in immunotherapies for cancer. Int J Mol Sci. 2019;20 (8):1822. doi:10.3390/ijms20081822

44. Moro K, Yamada T, Tanabe M, et al. Innate production of $\mathrm{T}(\mathrm{H}) 2$ cytokines by adipose tissue-associated c-Kit(+)Sca-1(+) lymphoid cells. Nature. 2010;463(7280):540-544. doi:10.1038/nature08636

45. Wilhelm C, Hirota K, Stieglitz B, et al. An IL-9 fate reporter demonstrates the induction of an innate IL-9 response in lung inflammation. Nat Immunol. 2011;12(11):1071-1077. doi:10.1038/ni.2133

46. Turner JE, Morrison PJ, Wilhelm C, et al. IL-9-mediated survival of type 2 innate lymphoid cells promotes damage control in helminth-induced lung inflammation. $J$ Exp Med. 2013;210 (13):2951-2965. doi:10.1084/jem.20130071

47. Krabbendam L, Bal SM, Spits H, et al. New insights into the function, development, and plasticity of type 2 innate lymphoid cells. Immunol Rev. 2018;286(1):74-85. doi:10.1111/imr.12708
48. Colonna M. Innate lymphoid cells: diversity, plasticity, and unique functions in immunity. Immunity. 2018;48(6):1104-1117. doi:10.1016/j.immuni.2018.05.013

49. Lim AI, Menegatti S, Bustamante J, et al. IL-12 drives functional plasticity of human group 2 innate lymphoid cells. J Exp Med. 2016;213(4):569-583. doi:10.1084/jem.20151750

50. Golebski K, Ros XR, Nagasawa M, et al. IL-1beta, IL-23, and TGF-beta drive plasticity of human ILC2s towards IL-17-producing ILCs in nasal inflammation. Nat Commun. 2019;10(1):2162. doi:10.1038/s41467-019-09883-7

51. Bartemes KR, Kephart GM, Fox SJ, et al. Enhanced innate type 2 immune response in peripheral blood from patients with asthma. J Allergy Clin Immunol. 2014;134(3):671-678 e4. doi:10.1016/j. jaci.2014.06.024

52. Winkler C, Hochdörfer T, Israelsson E, et al. Activation of group 2 innate lymphoid cells after allergen challenge in asthmatic patients. J Allergy Clin Immunol. 2019;144(1):61-69 e7. doi:10.1016/j. jaci.2019.01.027

53. Lao-Araya M, Steveling E, Scadding GW, et al. Seasonal increases in peripheral innate lymphoid type 2 cells are inhibited by subcutaneous grass pollen immunotherapy. J Allergy Clin Immunol. 2014;134 (5):1193-5 e4. doi:10.1016/j.jaci.2014.07.029

54. Robinette ML, Fuchs A, Cortez VS, et al. Transcriptional programs define molecular characteristics of innate lymphoid cell classes and subsets. Nat Immunol. 2015;16(3):306-317. doi:10.1038/ni.3094

55. Wilhelm C, Harrison OJ, Schmitt V, et al. Critical role of fatty acid metabolism in ILC2-mediated barrier protection during malnutrition and helminth infection. $J$ Exp Med. 2016;213(8):1409-1418. doi:10.1084/jem.20151448

56. Monticelli LA, Buck MD, Flamar A-L, et al. Arginase 1 is an innate lymphoid-cell-intrinsic metabolic checkpoint controlling type 2 inflammation. Nat Immunol. 2016;17(6):656-665. doi:10.1038/ ni. 3421

57. Klose CSN, Artis D. Innate lymphoid cells control signaling circuits to regulate tissue-specific immunity. Cell Res. 2020;30(6):475-491. doi:10.1038/s41422-020-0323-8

58. Boonpiyathad T, Tantilipikorn P, Ruxrungtham K, et al. IL-10producing innate lymphoid cells increased in house-dust-mite allergic rhinitis patients following immunotherapy. J Allergy Clin Immunol. 2020.

59. Trakarnsanga K, Wilson MC, Heesom KJ, et al. Secretory factors from OP9 stromal cells delay differentiation and increase the expansion potential of adult erythroid cells in vitro. Sci Rep. 2018;8 (1):1983. doi:10.1038/s41598-018-20491-1

\section{Publish your work in this journal}

The Journal of Asthma and Allergy is an international, peer-reviewed open-access journal publishing original research, reports, editorials and commentaries on the following topics: Asthma; Pulmonary physiology; Asthma related clinical health; Clinical immunology and the immunological basis of disease; Pharmacological interventions and new therapies. The manuscript management system is completely online and includes a very quick and fair peer-review system, which is all easy to use. Visit http://www.dovepress.com/testimonials.php to read real quotes from published authors. 